TRANSACTIONS OF THE

AMERICAN MATHEMATICAL SOCIETY

Volume 353, Number 1, Pages 117-132

S 0002-9947(00)02506-X

Article electronically published on August 21, 2000

\title{
CONNECTIVITY AT INFINITY FOR RIGHT ANGLED ARTIN GROUPS
}

\author{
NOEL BRADY AND JOHN MEIER
}

\begin{abstract}
We establish sufficient conditions implying semistability and connectivity at infinity properties for $\operatorname{CAT}(0)$ cubical complexes. We use this, along with the geometry of cubical $K(\pi, 1)$ 's to give a complete description of the higher connectivity at infinity properties of right angled Artin groups. Among other things, this determines which right angled Artin groups are duality groups. Applications to group extensions are also included.
\end{abstract}

\section{INTRODUCTION}

One of the oldest asymptotic invariants of discrete groups is the connectivity at infinity properties. At the lowest level there is the well known result of Freudenthal and Hopf that every finitely generated group is zero, one, two or infinitely ended. In terms of this taxonomy, the one-ended groups are certainly the least understood. The celebrated result of Stallings [St] reduced the study of infinitely ended groups to that of one-ended groups. The recent work on JSJ decompositions can be viewed as one attempt to better understand the collection of one-ended groups, but there is an older approach which asks about higher connectivity properties at infinity. For example, a finitely presented one-ended group $G$ is simply connected at infinity (roughly speaking) if loops near infinity in the Cayley graph can be filled by disks near infinity in the Cayley 2-complex. Simple connectivity at infinity has been particularly important in manifold theory; see, for example, Da 1 and $\S 0$ of BT. Assuming $G$ satisfies an appropriate finiteness condition, $G$ is $m$-connected at infinity if $n$-spheres near infinity in the universal cover of a $K(G, 1)$ can be filled by $(n+1)$-balls (near infinity) for all $-1 \leq n \leq m$. In GM 2 there is an analogue of the Freudenthal-Hopf result for $\pi_{1}$ at infinity. Notable recent examples of higher connectivity at infinity computations include those done for Coxeter groups ([Da 2]) and the group of outer automorphisms of free groups $([\mathrm{R},[\mathrm{V}]$, and most recently $[\overline{\mathrm{BF}})$.

Our basic technique for establishing connectivity at infinity properties works in the context of locally finite, $C A T(0)$ cubical complexes. Let $X$ be such a complex and let $v \in X^{(0)}$. If $w$ is a vertex of $X$, then $L k(w, X)$ can be thought of as

Received by the editors December 4, 1997 and, in revised form, February 5, 1999.

2000 Mathematics Subject Classification. Primary 20F36, 57M07.

Key words and phrases. Topology at infinity, right angled Artin groups, cubical complexes.

The first author thanks the Universitat Frankfurt for support during the summer of 1997 while part of this work was being carried out. He also acknowledges support from NSF grant DMS-9704417. The second author thanks Cornell University for hosting him while on leave from Lafayette College, and the NSF for the support of an RUI grant DMS-9705007. 
the space of directions based at $w$. In particular, the geodesic $[v, w]$ determines one direction in $L k(w, X)$ and we let $O p(w, v)$ denote the maximal subcomplex of $L k(w, X)$ composed of simplices which are further from $v$ than $w$. In $\S 4$ we establish

Corollary 4.2. Let $X$ be a locally finite, $C A T(0)$ cubical complex with $v \in X^{(0)}$. If the link of $v$ is $m$-connected, and if for each vertex $w \neq v, O p(w, v)$ is $m$-connected, then $X$ is $m$-connected at infinity and $\pi_{m+1}$-semistable.

We define $\pi_{m+1}$-semistable in $\S 2.1$; for now, think of the conditions " $m$-connected at infinity and $\pi_{m+1}$-semistable" as implying that the one point compactification of $X$ is locally $(m+1)$-connected.

We get complete results in the context of right angled Artin groups. Every finite flag complex $L$ has an associated infinite group $A_{L}$ whose generators are in one-toone correspondence with the vertices of $L$, and whose defining relations state that two generators commute if and only if they are joined by an edge in $L^{(1)}$. Each right angled Artin group $A_{L}$ has an associated $K\left(A_{L}, 1\right)$ constructed by gluing together tori; we denote this complex by $K_{L}$ and note that the universal cover $\widetilde{K_{L}}$ is a $\operatorname{CAT}(0)$ cubical complex.

Theorem A. Right angled Artin groups are homologically and homotopically semistable in all dimensions.

Theorem B. The group $A_{L}$ is m-connected ( $m$-acyclic) at infinity if and only if the link of a vertex $v \in \widetilde{K_{L}}$ is $m$-connected ( $m$-acyclic).

Since the complex $\widetilde{K_{L}}$, and the link of any vertex in $\widetilde{K_{L}}$, is determined by $L$, connectivity at infinity for right angled Artin groups is essentially determined by the topology of the defining complex. This can be made very explicit; in the following lemma, the condition "(-1)-acyclic" means "non-empty" and any lower terms are vacuous.

Lemma 5.1. The link of a vertex in $\widetilde{K_{L}}$ is $m$-acyclic if and only if $L$ is $m$-acyclic, and for any simplex $\sigma \subset L$, the link of $\sigma$ in $L$ is $(m-|\sigma|-1)$-acyclic. The link of a vertex in $\widetilde{K_{L}}$ is $m$-connected if, in addition to the conditions above, $L$ is simply connected.

In particular, combining Lemma 5.1 and Theorem B we have

Corollary 5.2. Let $L$ be a finite flag complex which is different from the 0- or 1-simplex. Then the right angled Artin group $A_{L}$ is simply connected at infinity if and only if $L$ is simply connected and contains no cut vertex.

A group $G$ is a duality group if there is an integer $n$ and a $G$-module $D$ such that $H^{i}(G, M) \approx H_{n-i}(G, D \otimes M)$ for all integers $i$ and $G$-modules $M$. Equivalently, it's a duality group if its cohomology with group ring coefficients is torsion free and concentrated in dimension $n$.

Theorem C. A right angled Artin group $A_{L}$ is a duality group if and only if $L$ is Cohen-Macaulay.

Recall that an $n$-dimensional simplicial complex $L$ is Cohen-Macaulay if its reduced homology is concentrated in dimension $n$ and for each simplex $\sigma \subset L$, the reduced homology of the link of $\sigma$ in $L$ is concentrated in dimension $n-|\sigma|-1$.

We note that a right angled Artin group $A_{L}$ is a Poincaré duality group $\Leftrightarrow L$ is a simplex and hence $A_{L}$ is free abelian. The argument uses Strebel's theorem that 
says any infinite index subgroup $H$ of a Poincaré duality group $G$ must have strictly smaller cohomological dimension than that of $G[\underline{\mathrm{Sr}}$. However, the dimension of $A_{L}$ is the dimension of its largest free abelian subgroup, and maximal free abelian subgroups are of infinite index in $A_{L}$ unless $A_{L}$ is itself free abelian.

Our original motivation for studying connectivity at infinity for right angled Artin groups arose from our efforts to understand work relating connectivity at infinity to finiteness properties of infinite index normal subgroups (see for example $[\mathrm{H}], \mathrm{J}]$, GM 1], and [Mi 2]). For example, Houghton and Jackson (independently) showed that if $1 \rightarrow K \rightarrow G \rightarrow L \rightarrow 1$ is a short exact sequence of finitely presented groups with $L$ one-ended, then $G$ is simply-connected at infinity. Profio pointed out that one cannot reduce the assumption that the kernel is finitely presented to finitely generated $[\mathrm{P}$, and Mihalik has shown that this reduction cannot occur even if one assumes very strong connectivity at infinity properties for the quotient Mi 3. In both examples the extension $G$ is a right angled Artin group.

The finiteness properties of normal subgroups above the commutator of a right angled Artin group are understood by work in [MMV], and connectivity at infinity properties of $A_{L}$ are understood by Theorem $\mathrm{B}$ above. In the last section we combine these results to construct several illuminating examples which extend the Profio-Mihalik type examples. In particular, there is a short exact sequence $1 \rightarrow K \rightarrow A_{L} \rightarrow \mathbb{Z}^{n} \rightarrow 1$ where the quotient is free abelian (of arbitrary finite rank), and $K$ is $F P_{\infty}$ and one-ended, but $A_{L}$ is not simply connected at infinity.

We thank Ken Brown, Mike Davis, Ross Geoghegan and Mike Mihalik for answering our many questions as we worked on this material. In particular we note that Mike Davis provided us with an alternate proof of the homological version of Theorem B. Davis's argument starts by using a clever trick showing that every right angled Artin group embeds as a finite index subgroup of a right angled Coxeter group. One can then apply his theorem on cohomology with group ring coefficients for Coxeter groups [Da 2] to get the homology at infinity result.

\section{Background and Definitions}

Here we collect information in three subsections, with further references in each subsection for the reader unfamiliar with the material. Our presentation is rather terse, and is intended primarily to establish notational conventions.

2.1. Semistability and Connectivity at Infinity. A group $G$ is $F_{m}$ if there is a $K(G, 1)$ whose $m$-skeleton is finite; $G$ is $F P_{m}$ if $\mathbb{Z}$ thought of as a trivial $\mathbb{Z} G$-module has a projective resolution that is finite through dimension $m$. The action of $G$ on the universal cover of a $K(G, 1)$ provides such a resolution, so $F_{m} \Rightarrow F P_{m}$ but the converse is false for all $m>1[\mathrm{BB}]$. Let $G$ be a $F_{m+1}$ group, and let $X$ be the universal cover of a $K(G, 1)$ whose $(m+1)$-skeleton is finite. Then $G$ is $m$-connected at infinity if given any compact set $C \subset X$ there is a compact set $D \subset X$ such that any map $\varphi: S^{n} \rightarrow X-D$ extends to a map $\hat{\varphi}: B^{n+1} \rightarrow X-C$ for all $0 \leq n \leq m$. Similarly, still assuming $G$ is $F_{m+1}, G$ is $m$-acyclic at infinity if any $n$-cycle whose support is outside of $D$ is the boundary of an $(n+1)$-chain with support outside of $C$ for all $-1 \leq n \leq m$. (For background on the $F_{m}$ and $F P_{m}$ properties, see $[\mathrm{Br}$; see also G] for a detailed discussion of homotopy and homology at infinity.)

In addition to $m$-connectivity at infinity, one can define homotopy and homology groups at infinity. Let $C_{0} \subset C_{1} \subset \cdots \subset C_{n} \subset \cdots$ be a nested properly increasing sequence of compact subcomplexes that exhaust $X$. Since we are assuming that $X$ 
is one-ended, we will also assume that for all $i, X-C_{i}$ consists of one component. Then there are induced sequences of homotopy and homology groups

$$
\pi_{m}\left(X-C_{0}\right) \leftarrow \pi_{m}\left(X-C_{1}\right) \leftarrow \pi_{m}\left(X-C_{2}\right) \leftarrow \cdots
$$

and

$$
H_{m}\left(X-C_{0}\right) \leftarrow H_{m}\left(X-C_{1}\right) \leftarrow H_{m}\left(X-C_{2}\right) \leftarrow \cdots
$$

One needs to take care of basepoints with the homotopy groups. The easiest way to do this is to let $\rho:[0, \infty) \rightarrow X$ be a proper ray where $\rho([n, \infty)) \subset X-C_{n}$ for all $n$. One can then let $\rho(n)$ be the basepoint for $\pi_{m}\left(X-C_{n}\right)$, and use the change of basepoint isomorphism induced by the path $\rho([n, n+1])$ to identify $\pi_{m}\left(X-C_{n}, \rho(n)\right)$ with $\pi_{m}\left(X-C_{n}, \rho(n+1)\right)$; thus the map

$$
\pi_{m}\left(X-C_{n+1}, \rho(n+1)\right) \rightarrow \pi_{m}\left(X-C_{n}, \rho(n+1)\right)
$$

induced by inclusion can be extended to a map $\pi_{m}\left(X-C_{n+1}, \rho(n+1)\right) \rightarrow$ $\pi_{m}\left(X-C_{n}, \rho(n)\right)$. If any two proper rays in $X$ are properly homotopic, then the choice of proper base ray $\rho$ is not important. In this case you can define $\pi_{m}^{\infty}(X)$ to be the inverse limit of the sequence of homotopy groups. Likewise $H_{m}^{\infty}(X)$ is defined to be the inverse limit of the homology groups.

The induced system of homotopy or homology groups is semistable if for every $n$ there is an $f(n) \geq n$ such that the image of $\pi_{m}\left(X-C_{k}\right) \rightarrow \pi_{m}\left(X-C_{n}\right)$ (or $H_{m}\left(X-C_{k}\right) \rightarrow H_{m}\left(X-C_{n}\right)$ ) is independent of $k$ for $k \geq f(n)$. In the case of homotopy groups, this is equivalent to being able to freely homotope maps $f: S^{m} \rightarrow\left(X-C_{f(n)}\right)$ to maps $f^{\prime}: S^{m} \rightarrow\left(X-C_{k}\right)$ (for any $k \geq f(n)$ ) by a homotopy missing $C_{n}$. Homological semistability is similar except that one finds $m$-cycles in $X-C_{k}$ homologous in $X-C_{n}$ to $m$-cycles in $X-C_{f(n)}$. Mihalik proved that the minimal property necessary to have $\pi_{1}^{\infty}(X)$ is well defined, that is, that proper rays are properly homotopic, is actually equivalent to $\pi_{1}$-semistability [Mi 1]. For further background see [MS], GM 2] and the references cited there.

Instead of complements we will frequently need to use closed complements, that is, if $L \subset K$, then set $L-K=\overline{L-K}$. When discussing connectivity at infinity, one usually uses an exhausting sequence of compact (in particular closed) sets, but even in this context our using closed complements will present no technical difficulties. Although our notation is reminiscent of the "CW complement" of [G] these closures of complements are different.

2.2. Cubical Complexes. A cubical complex is a complex constructed out of cubes where, just like simplicial complexes, if two cubes intersect, then they do so in a proper face. One can give a cubical complex a piecewise Euclidean metric by assigning the metric of the unit Euclidean $n$-cube to each $n$-cell. One can identify $L k(v, X)$ with the space of all directions based at $v$, or equivalently, one can think of $L k(v, X)$ as the metric unit sphere about $v$. Thought of this way, $L k(v, X)$ inherits a natural (piecewise spherical) simplicial structure. A piecewise Euclidean cubical complex $X$ is "non-positively curved" (more accurately "CAT(0)") if and only if $X$ is simply connected and the links of all vertices of $X$ are flag complexes. Recall that a flag complex is a simplicial complex $L$ that is determined by its one-skeleton; given any complete graph $C \subset L^{(1)}$ there is a simplex $\sigma \subset L$ with $\sigma^{(1)}=C$. For general background on $\mathrm{CAT}(0)$ complexes see $[\mathrm{BH}]$. 
We will use pairs when describing the various subcomplexes encountered throughout this paper. For example, if $\mathrm{K}$ is a subcomplex of a complex $L$ (cubical or simplicial), let $N b^{1}(K, L)=\{$ cells $\sigma$ of $L \mid \sigma \cap K \neq \emptyset\}$ be the first cellular neighborhood of $K$ in $L$. Similarly, let $N b^{m}(K, L)$ be defined to be $N b^{1}\left(N b^{m-1}(K, L), L\right)$ for $m \geq 2$. If $L$ is a simplicial complex, we use $L k(\sigma, L)$ to denote the link the simplex $\sigma$ in $L$. Note that the star $S t(\sigma, L)$ is isomorphic to the join $\sigma * L k(\sigma, L)$. If $v$ is a vertex in a cubical complex, then $L k(v, X)$ denotes the link of $v$ in $X$.

Remark 2.1. Let $v$ be a vertex in a cubical complex $X$. We will have occasion to move between the link $L k(v, X)$ and the boundary $\partial N b^{1}(v, X)$ of the first cellular neighborhood of $v$. Radial projection from $v$ induces a homeomorphism $\varrho: L k(v, X) \rightarrow \partial N b^{1}(v, X)$. In particular, the cubulation of $L k(v, X)$ is isomorphic (as a cubical complex) to $\partial N b^{1}(X)$. (The reader unfamiliar with the notion of cubulation can take the above map as the definition.)

Proofs of the following three lemmas can be found in $\mathrm{BB}$.

Lemma 2.2. If $v$ is a vertex of $\widetilde{K_{L}}$ and $Q$ is a cube in $\widetilde{K_{L}}$, then $d(v, Q)$ is attained at a unique vertex of $Q$.

Lemma 2.3. Let $M \subset \widetilde{K_{L}}$ be a subcomplex. If $L k(v, M) \subset L k\left(v, \widetilde{K_{L}}\right)$ is a full subcomplex for each vertex $v \in M$, then $M$ is convex in $\widetilde{K_{L}}$.

As mentioned above, the links $L k(v, X)$ inherit an "all right" metric structure. That is, the simplices are isomorphic to the intersection of the unit sphere with the cone determined by the positive coordinate axes in $\mathbb{R}^{n}$.

Lemma 2.4. If $\sigma_{1}, \sigma_{2}$ are simplices in a finite, all-right, piecewise spherical flag complex satisfying $\sigma_{1} \cap \sigma_{2}=\emptyset$, then $d\left(\sigma_{1}, \sigma_{2}\right) \geq \pi / 2$.

Remark and Definition 2.5. Given a vertex $v$ in a CAT(0) cubical complex $X$, for each vertex $w \in X$ the geodesic $[v, w]$ determines a point in $L k(w, X)$. Let $\sigma_{v} \subset$ $L k(w, X)$ be the minimal simplex containing this point. We define the opposite part of $L k(w, X)$ (with respect to $v$ ) to be $O p(w, v)=L k(w, X){ }_{c} N b^{1}\left(\sigma_{v}, L k(w, X)\right)$. The subcomplex $O p(w, v)$ determines a subcomplex $\varrho(O p(w, v)) \subset \partial N b^{1}(w, X)$. Using Lemma 2.4 one can show that a cube $\square \subset N b^{1}(w, X)$ corresponds to a simplex in $O p(w, v)$ if and only if $w$ is the unique closest point to $v$ in $\square$. Throughout this paper we will be replacing collections of cells containing a vertex $w$ by cells in $O p(w, v)$. Because all the cells in $O p(w, v)$ are further from $v$ than $w$, this procedure will move collections of cells in $X$ away from $v$.

2.3. Right Angled Artin Groups. Given a flag complex $L$, the associated right angled Artin group $A_{L}$ has generators in one-to-one correspondence with the vertices of $L$, and defining relations stating that two generators commute when the corresponding vertices are adjacent in the 1-skeleton of $L$. Since these groups really only depend on the one-skeleton of $L$, they are also called "graph groups."

One can create a non-positively curved $K\left(A_{L}, 1\right)$ by gluing together tori. For each simplex $\sigma \subset L$ let $T_{\sigma}$ be a $(|\sigma|+1)$-torus, where $|\sigma|$ is the dimension of $\sigma$. Then $K_{L}$ is the space

$$
K_{L} \equiv\left[\coprod_{\sigma \subset L} T_{\sigma}\right] /\left\{T_{\sigma} \cap T_{\sigma^{\prime}}=T_{\sigma \cap \sigma^{\prime}}\right\}
$$



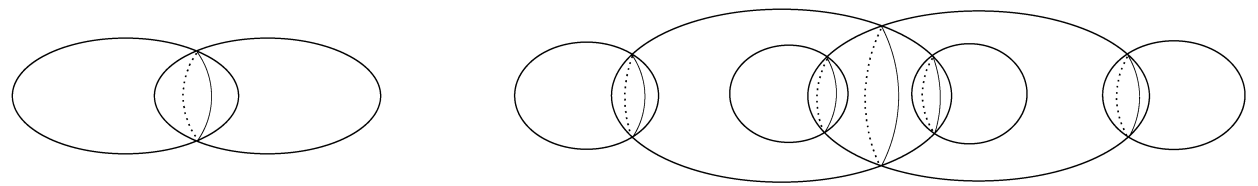

Figure 1. The boundaries of the first two neighborhoods of $v$ in $\widetilde{K_{L}}$

where $T_{\emptyset}$ is the basepoint. The universal cover $\widetilde{K_{L}}$ of $K_{L}$ is a contractible, $\operatorname{CAT}(0)$ cubical complex. The link of the single vertex in $K_{L}$ is denoted $S_{L}$. It can be constructed directly from the defining complex $L$. For each vertex $v \in L$ let $S_{v}$ be a copy of the zero sphere with vertices named $\left\{v^{-}, v^{+}\right\}$. For any simplex $\sigma \subset L$ let $S_{\sigma}=S_{v_{0}} * \cdots * S_{v_{k}}$ where $\sigma^{(0)}=\left\{v_{0}, \ldots, v_{k}\right\}$. The link $S_{L}$ can then be described as

$$
S_{L} \simeq\left[\coprod_{\sigma \subset L} S_{\sigma}\right] /\left\{S_{\sigma} \cap S_{\sigma^{\prime}}=S_{\sigma \cap \sigma^{\prime}}\right\}
$$

where $S_{\emptyset}=\emptyset$. Note that the subcomplex induced by $\left\{v_{i}^{+} \in S_{L} \mid v_{i} \in L\right\}$ is a copy of $L$ in $S_{L}$. Also notice that given any flag complex $K$ one can construct the complex $S_{K}$ without reference to any ambient collection of tori.

In $\S 3$ we will be working with the boundaries of the iterated cellular neighborhoods of a given base vertex $v$ in $\widetilde{K_{L}}$. For example, if $L$ is the union of two triangles joined along a common side, then $A_{L} \simeq \mathbb{Z}^{3} *_{\mathbb{Z}^{2}} \mathbb{Z}^{3}$ and the boundaries of the first two cellular neighborhoods of $v$ are (topologically) 2-spheres joined along 1-spheres as in Figure 1. In order to familiarize themselves with these $K(\pi, 1)$ 's, we recommend that the interested reader verify the accuracy of Figure 1 before moving on to later sections.

An $n$-flat in $\widetilde{K_{L}}$ is an isometrically embedded copy of $\mathbb{R}^{n}$ in $\widetilde{K_{L}} ;$ sheets are flats in $\widetilde{K_{L}}$ which are components of preimages (under the universal covering map) of tori in $K_{L}$. For example, the sheets in the standard tree associated to the free group $F_{2}$ are precisely the 'horizontal' and 'vertical' lines.

For further background see $[\mathrm{BB}]$ and $[\mathrm{MV}]$.

\section{Convexity and Semistability}

In this section we will exhaust the spaces $\widetilde{K_{L}}$ using the cellular neighborhoods of a chosen base point. The first step is to establish that these subcomplexes are convex.

Lemma 3.1. For any flag complex $L$ and any $n \geq 1, N b^{n}\left(v, \widetilde{K_{L}}\right)$ is convex in $\widetilde{K_{L}}$.

Proof. We argue by induction on $n$, using Lemma 2.3 to establish convexity.

Base Case: We check that $N b^{1}\left(v, \widehat{K_{L}}\right)$ is convex by verifying that for each vertex $w \in N b^{1}\left(v, \widetilde{K_{L}}\right)$ the link $L k\left(w, N b^{1}(v)\right)$ is a full subcomplex of the link $L k\left(w, \widetilde{K_{L}}\right)=$ $S_{L}$. If $w=v$ is the basepoint, then $L k\left(w, N b^{1}(v)\right)=S_{L}$. If $w$ is not the basepoint, then the geodesic $[v, w]$ determines a point of $L k\left(w, \widetilde{K_{L}}\right)$. Let $\sigma$ be the unique minimal simplex of $L k\left(w, \widetilde{K_{L}}\right)$ containing this point. There is a natural map $\Phi$ from $S_{L}$ onto $L$ given by sending each vertex to the corresponding positive vertex $v_{i}^{ \pm} \mapsto v_{i}^{+}$. The group $\mathbb{Z}_{2}^{|V(L)|}$ acts on $S_{L}$ and the map $\Phi$ can be identified with the 
projection onto the orbit space. Note that

$$
\operatorname{Lk}\left(w, N b^{1}(v)\right) \simeq S t\left(\sigma, S_{L}\right)
$$

which is full in $S_{L}$, since $S_{L}$ is a flag complex.

Inductive step: Given that $N b^{k}\left(v, \widetilde{K_{L}}\right)$ is convex, we wish to show that $N b^{k+1}\left(v, \widetilde{K_{L}}\right)$ is also convex. Any vertex of $N b^{k+1}\left(v, \widetilde{K_{L}}\right)$ which is contained in $N b^{k}\left(v, \widetilde{K_{L}}\right)$ has all of $S_{L}$ as its link, so it suffices to consider a vertex $w$ belonging to $N b^{k+1}\left(v, \widehat{K_{L}}\right)-$ $N b^{k}\left(v, \widetilde{K_{L}}\right)$. There exists a unique vertex $x \in N b^{k}\left(v, \widetilde{K_{L}}\right)$ so that

$$
d(x, w)=d\left(w, N b^{k}\left(v, \widetilde{K_{L}}\right)\right) .
$$

Such a point exists because $N b^{k}\left(v, \widetilde{K_{L}}\right)$ is compact; it is unique by the $\operatorname{CAT}(0)$ inequality and the fact that $N b^{k}\left(v, \widetilde{K_{L}}\right)$ is convex (by induction); and the point $x$ is a vertex by Lemma 2.2. As above, let $\sigma$ denote the minimal simplex of $L k\left(w, \widetilde{K_{L}}\right)$ determined by $[x, w]$. We claim that

$$
\operatorname{Lk}\left(w, N b^{k+1}\left(v, \widetilde{K_{L}}\right)\right) \simeq \operatorname{St}\left(\sigma, S_{L}\right)
$$

Note that $\supset$ is clear. This is just the contribution from cubes which contain both $x$ and $w$. So we only have to show that every cube which intersects $N b^{k}$ and contains $w$ must also contain $x$. Suppose to the contrary that $Q$ is a cube in $\widetilde{K_{L}}$ such that $x \notin Q, w \in Q$, and $Q \cap N b^{k} \neq \emptyset$. Then let $x^{\prime}$ be the vertex of $Q \cap N b^{k}$ which is closest to $x$, and let $Q^{\prime}$ be the minimal cube at $x^{\prime}$ determined by the terminal segment of $\left[x, x^{\prime}\right]$. Consider the geodesic triangle $\triangle x x^{\prime} w$. Now $x^{\prime} \neq x$ by assumption, and so $d(x, w)<d\left(x^{\prime}, w\right)$ by choice of $x$. Thus $\measuredangle w x^{\prime} x<\pi / 2$, and therefore the simplices $\sigma, \sigma^{\prime}$ of $L k\left(x^{\prime}, \widehat{K_{L}}\right)$ determined by $Q$ and $Q^{\prime}$ respectively are within $\pi / 2$ of each other in the spherical metric. By Lemma 2.4, $\sigma \cap \sigma^{\prime} \neq \emptyset$ and so the cubes $Q$ and $Q^{\prime}$ share a common face (of dimension at least one) at $x^{\prime}$. But this face is contained in $Q \cap N b^{k}$ and so the distance from $w$ to this face is not attained at $x^{\prime}$. This contradicts the choice of $x^{\prime}$.

Since the cellular neighborhoods are convex, nearest point retraction is a deformation retraction from the closed complement $\widetilde{K_{L}}-\frac{N}{c}-b^{k}\left(v, \widetilde{K_{L}}\right)$ onto $\partial N b^{k}(v)$. It follows that

$$
\pi_{m}\left(\widetilde{K_{L}}-\frac{N}{c} b^{k}\left(v, \widetilde{K_{L}}\right)\right) \simeq \pi_{m}\left(\partial N b^{k}\left(v, \widetilde{K_{L}}\right)\right)
$$

for all $m$. We can now restate Theorem A more precisely:

Theorem A. Let $\widetilde{K_{L}}$ be the universal cover of the standard $K(\pi, 1)$ for $A_{L}$. Then all of the inclusion induced homomorphisms in the sequences

$$
\pi_{n}\left(\widetilde{K_{L}}-{ }_{c} N b^{1}(v)\right) \leftarrow \cdots \leftarrow \pi_{n}\left(\widetilde{K_{L}}-\frac{}{c} N b^{k}(v)\right) \leftarrow \pi_{n}\left(\widetilde{K_{L}}-{ }_{c}-N b^{k+1}(v)\right) \leftarrow \cdots
$$

and

$$
\left.H_{n}\left(\widetilde{K_{L}}-N b^{1}(v)\right) \leftarrow \cdots \leftarrow H_{n} \widetilde{\left(\bar{K}_{L}\right.}-N b_{c}^{k}(v)\right) \leftarrow H_{n}\left(\widetilde{K_{L}}-N b_{c}^{k+1}(v)\right) \leftarrow \cdots
$$

are surjections. In particular, $\widetilde{K_{L}}$ is homologically and homotopically semistable in all dimensions. 


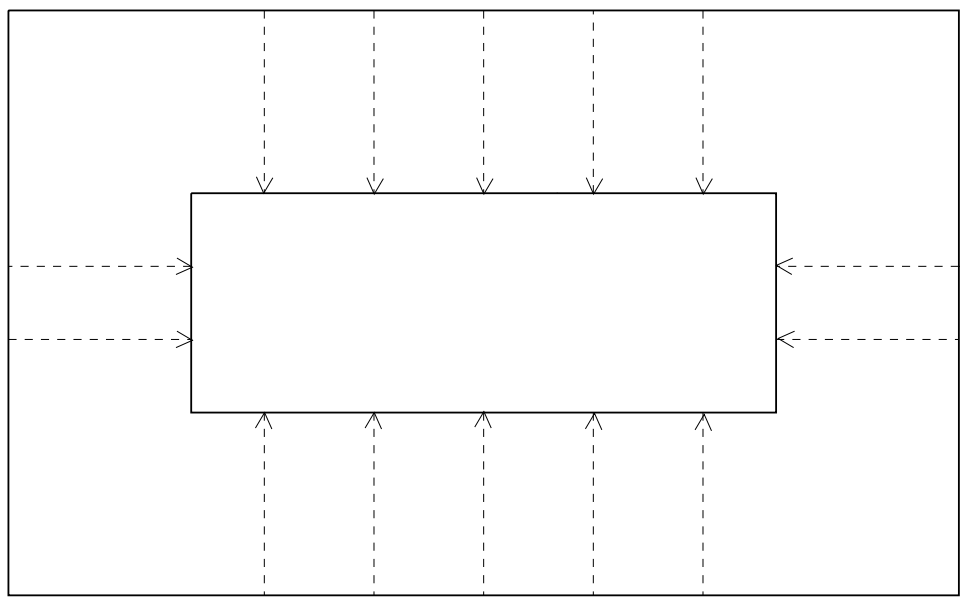

FIGURE 2. The free homotopy inside a 2-sheet

Proof. There is a copy of $\partial N b^{k}(v)$ contained in $\left.\widetilde{\left(K_{L}\right.}-N b^{k+1}(v)\right)$, which is homotopic to $\partial N b^{k}(v)$ in $\widetilde{K_{L}}-N b_{c}^{k}\left(v, \widetilde{K_{L}}\right)$. The copy is constructed by examining sheets passing through vertices of $N b^{k-1}(v)$. Given an $m$-sheet $S h \ni w$ with $w \in N b^{k-1}(v)$, we know that $S h \cap N b^{k}(v)$ is a convex subcomplex since it is the intersection of convex subcomplexes. The intersection is actually an $m$-dimensional rectangle $\simeq \prod_{i=1}^{m}\left[a_{i}, b_{i}\right]$ (where $a_{i}<b_{i}$ are both in $\mathbb{Z}$ ) since $w \in N b^{k-1}(v)$ hence the cellular neighborhood of $w$ in $N b^{k}(v)$ is $N b^{1}(w)$.

Let $d$ be any positive integer. Let $C=\bigcup \prod_{i=1}^{m}\left[a_{i}-d, b_{i}+d\right]$ where the union is taken over all sheets containing vertices of $N b^{k-1}(v)$. We claim that $\partial C$ is freely homotopic to $\partial N b^{k}(v)$ in $\widetilde{K_{L}}-N b_{c}^{k}\left(v, \widetilde{K_{L}}\right)$. We build the homotopy inductively on sheets beginning with the 1-sheets. Each 1-sheet $S h$ intersects $C$ in an interval $\left[a_{i}-d, b_{i}+d\right]$, hence there is a natural map in the sheet from $S h \cap \partial C$ to $S h \cap \partial N b^{k}(v)$. If $S h$ is a 2 -sheet, then the homotopy has already been defined on certain vertices in the boundary, and we complete the definition by sending the corners to the corners as in Figure 2.

One constructs the homotopy by continuing in this manner. Since the intersection of two sheets is a sheet, and our definition begins in lowest dimension, the map is well-defined. By choosing $d$ sufficiently large, one can find a copy of $\partial N b^{k}(v)$ in $\partial N b^{n}(v)$ for any $n \geq k$.

\section{Connectivity at Infinity}

In this section we establish sufficient conditions implying that a $C A T(0)$ cubical complex is $m$-connected at infinity. We then apply this to establish our Theorem B. The argument is by induction, and the base case establishes that our discussion is well-defined. That is, that our spaces are one-ended, and that proper rays are properly homotopic. In the case of right angled Artin groups (with $L$ connected) this was already known (see Theorem 1.4 of [Mi 2] or Corollary 5.4 of [Me]), but the argument here is direct and motivates our later work. 
Proposition 4.1. Let $X$ be a locally finite, $C A T(0)$ cubical complex with $v \in X^{(0)}$.

(i) If the link of $v$ is connected, and if for each vertex $w \neq v, O p(w, v)$ is connected, then $X$ is one-ended and $\pi_{1}$-semistable.

(ii) If the link of $v$ is m-acyclic, and if for each vertex $w \neq v, O p(w, v)$ is $m$-acyclic, then $X$ is homologically $m$-connected at infinity and $H_{m+1}$-semistable.

(iii) If the link of $v$ is 1-connected, and if for each vertex $w \neq v, O p(w, v)$ is 1-connected, then $X$ is simply connected at infinity.

In the proof we'll actually show that the complements are 1-connected or $m$ acyclic, hence we get the following corollary using the standard Hurewicz theorem. Even without the fact that the complements are well behaved the corollary would follow by a pro-Hurewicz theorem as in $\mathrm{MS}$.

Corollary 4.2. If the link of $v$ is $m$-connected, and if for each vertex $w \neq v$, $O p(w, v)$ is $m$-connected, then $X$ is $m$-connected at infinity and $\pi_{m+1}$-semistable.

Proof of Proposition 4.1. The arguments in all three cases are fairly similar, and we treat them in turn. In each case the filtration will be by metric balls

$$
B_{r}(v)=\{x \in X \mid d(v, x) \leq r\} .
$$

Proof of (i). To establish that $X$ is one-ended, we actually prove that $X-B_{r}(v)$ is path connected for any $r$. Let $w_{1}$ and $w_{2}$ be two vertices in $X-B_{r}(v)$, and let $p$ be any path in $X$ connecting $w_{1}$ to $w_{2}$. Since $L k(v, X)$ is connected, we can homotope $p$ to an edge path that misses $v$. Next, let $z$ be a point on $p$ that is of minimal distance $D$ to $v$. By Lemma $2.2 z$ is a vertex, and if $d(z, v)>r$, then $p$ is already contained in $X-B_{r}(v)$. Otherwise, we replace each subpath $\left[v_{i-1}, z, v_{i+1}\right]$ in $p$ by a path $\left[v_{i-1}, w_{i_{0}}, w_{i_{1}}, \ldots, w_{i_{l}}, v_{i+1}\right]$ contained in the portion of $\partial N b^{1}(z)$ determined by $O p(z, v)$. Such a path exists because $O p(z, v)$ is connected and $v_{i-1}$ and $v_{i+1}$ both determine points in $O p(z, v)$ because $z$ is of minimal distance to $v$. Continuing in this manner we replace all vertices of $p$ that are a distance $D$ from $v$ by vertices which are further from $v$. If this new edge path is still not outside of $B_{r}(v)$, we repeat the process. Note that, since the end points are outside of $B_{r}(v)$, they remained fixed throughout this process. Further, since each substitution takes place inside of $S t(z, X)$, a contractible subcomplex, we have actually described a fixed end point homotopy from $p$ to a path in $X-B_{r}(v)$.

To see that $X$ is semistable, and hence, that the groups $\pi_{1}^{\infty}(X)$ are well-defined, let $\rho_{0}$ and $\rho_{1}$ be two proper rays, that is, proper maps from $[0, \infty)$ to $X$. We will assume these are edge path rays where $\rho_{i}(n) \in X^{(0)}$ for all $n$ in $\mathbb{Z}_{\geq 0}$. Let $\gamma:[0,1] \rightarrow X$ be an edge path connecting $\rho_{0}(0)$ to $\rho_{1}(0)$. As in the argument above, after a finite number of local homotopies, we may assume that $\rho_{0}, \rho_{1}$ and $\gamma$ all miss $v$.

We define a proper homotopy $H:[0, \infty) \times I \rightarrow X$ between $\rho_{0}$ and $\rho_{1}$ in a piecewise fashion. Since the $\rho_{i}$ are proper, there is an increasing function $f: \mathbb{Z}_{\geq 0} \rightarrow \mathbb{Z}_{\geq 0}$ such that $f(0)=0$ and for each $n, d\left(\rho_{i}(x), v\right)>n$ for all $x \geq f(n)$. Define a sequence of homotopies $H_{n}:[f(n), f(n+1)] \times I \rightarrow X$ inductively by first describing what $H_{n}$ does to the top, bottom and left-hand edges of its domain by

$$
\begin{aligned}
\left.H_{n}\right|_{[f(n), f(n+1)] \times\{0\}} & =\left.\rho_{0}\right|_{[f(n), f(n+1)]}, \\
\left.H_{n}\right|_{[f(n), f(n+1)] \times\{1\}} & =\left.\rho_{1}\right|_{[f(n), f(n+1)]}, \\
\left.H_{n}\right|_{\{f(n)\} \times[0,1]} & =\left.H_{n-1}\right|_{\{f(n)\} \times[0,1]},
\end{aligned}
$$


where $\left.H_{0}\right|_{\{0\} \times[0,1]}=\gamma$. Thus the bottom and top edges are determined by sections of the edge paths $\rho_{i}$ and the left-hand edge is the right-hand edge of the previously defined homotopy.

The entire homotopy $H_{n}$ is constructed by gluing together the local homotopies described in the previous argument. The maps defined on the top, bottom and left-hand edges of the domain of $H_{n}$ describe an edge path connecting $\rho_{0}(f(n+1))$ to $\rho_{1}(f(n+1))$. By the definition of $f$ the end points are outside of $B_{n+1}$ and $\rho_{i}([f(n), f(n+1)]) \subset X-B_{n}(v)$; by induction $H_{n}(\{f(n)\} \times[0,1])$ is also in $X-B_{n}(v)$. By applying the local homotopies from the previous argument, we get a fixed end point homotopy converting this edge path to an edge path connecting $\rho_{0}(f(n+1))$ to $\rho_{1}(f(n+1))$ which is outside of $B_{n+1}(v)$. The concatenation of these local maps gives $H_{n}$. Glueing together the $H_{n}$ 's then forms the homotopy $H$; it is proper because the original rays $\rho_{i}$ were proper and because the local homotopies increase the distance from the base vertex $v$.

Proof of (ii). Let $z$ be an $m$-cycle with $\operatorname{supp}(z) \subset X-B_{r}(v)$. Because $X$ is contractible, there is some $(m+1)$-chain $c$ with $\partial c=z$. If the vertex $v \in \operatorname{supp}(c)$, then $\partial\left(\left.c\right|_{N b^{1}(v, X)}\right)$ is an $m$-cycle, hence there is an $(m+1)$-chain $e$ with support in $\partial N b^{1}(v, X)$ with $\partial e=\partial\left(\left.c\right|_{N b^{1}(v, X)}\right)$. (We are using the natural identification of the cubulation of $L k(v, X)$ with $\partial N b^{1}(v, X)$.) Note that if $c^{\prime}=c-\left.c\right|_{N b^{1}(v, X)}+e$, then $\partial c^{\prime}=\partial c=z$ and $v \notin \operatorname{supp}\left(c^{\prime}\right)$.

We use similar local replacements to continue pushing the chain $c^{\prime}$ away from $v$. Let $w \in \operatorname{supp}\left(c^{\prime}\right)$ be a vertex achieving the minimal distance from $v$ to $\operatorname{supp}\left(c^{\prime}\right)$. Note that $\left.c^{\prime}\right|_{\partial N b^{1}(w, X)}$ determines an $m$-cycle supported in $O p(w, v)$. By hypothesis there is an $(m+1)$-chain $e^{\prime}$ with $\operatorname{supp}\left(e^{\prime}\right) \subset O p(w, v)$ and $\partial e^{\prime}=\partial\left(\left.c^{\prime}\right|_{N b^{1}(w, X)}\right)$. The new $(m+1)$-chain $c^{\prime \prime}=c^{\prime}-\left.c^{\prime}\right|_{N b^{1}(w, X)}+e^{\prime}$ again has boundary equal to $z$. Continuing in this manner we can convert $c$ to a chain whose support is outside of $B_{r}(v)$ and whose boundary is $z$.

Note that each of these local replacements occur inside the star of some vertex; since stars are contractible, this argument can be modified in a straightforward manner to show that any $(m+1)$-chain in $X-B_{r}(v)$ is homologous to an $(m+1)$ chain in $X-B_{r^{\prime}}(v)$ for any $r^{\prime} \geq r$. It follows that $X$ is homologically $(m+1)$ semistable.

Proof of (iii). Let $p$ be a closed edge path in $X-B_{r}(v)$, and let $f: D \rightarrow X^{(2)}$ be a van Kampen diagram for $p$, based at some vertex of $p$. If there is a vertex $a \in D$ with $f(a)=v$, then because we are working with a van Kampen diagram, $f\left(\partial N b^{1}(a, D)\right) \subset \partial N b^{1}(f(a)=v, X)$. Since $v \notin p, \partial N b^{1}(a, D)$ is a circle; because $L k(v, X)$ is simply connected, there is a van Kampen diagram $f_{a}: D_{a} \rightarrow X$ for the edge path given by $f\left(\partial N b^{1}(a, D)\right)$. By replacing $N b^{1}(a, D)$ by this new van Kampen diagram, we have removed one vertex mapping to $v$. In this process parts of $\partial N b^{1}(a, D)$ may become identified, but we are still left with a van Kampen diagram for $p$. Continuing in this manner we may assume that $f(D) \cap v=\emptyset$.

In a similar manner, we may replace neighborhoods of vertices $b$ in $D$ whose image $f(b)$ minimizes the distance from $f(D)$ to $v$ by van Kampen diagrams arising from $O p(f(b), v)$. Repeated applications of these local homotopies yields a van Kampen diagram whose image is in $X-B_{r}(v)$.

We now turn to the proof of Theorem B from the introduction. 
$\Rightarrow$ : Assume $S_{L}$ is not $m$-connected $\left(m\right.$-acyclic). Then $\partial N b^{1}\left(v, \widetilde{K_{L}}\right)$ is not $m$ connected ( $m$-acyclic) and so $\pi_{m}^{\infty}\left(\widetilde{K_{L}}\right) \neq\{1\}\left(H_{m}^{\infty}\left(\widetilde{K_{L}}\right) \neq 0\right)$ by Theorem A.

$\Leftarrow$ : This is a simple corollary to Proposition 4.1 using the following lemma.

Lemma 4.3. Let $\sigma$ be any simplex in $S_{L}$. If $S_{L}$ is $m$-connected (m-acyclic), then the closure of the complement of $N b^{1}(\sigma), S_{L} \underset{c}{c} N b^{1}\left(\sigma, S_{L}\right)$, is also m-connected (m-acyclic).

Proof. Without loss of generality we will assume that $\sigma^{(0)}=\left\{v_{0}^{-}, \ldots, v_{k}^{-}\right\}$. Define a map $\psi_{\sigma}: S_{L} \rightarrow S_{L}-N b^{1}\left(\sigma, S_{L}\right)$ starting with the 0-skeleton: $\psi_{\sigma}\left(v^{ \pm}\right)=v^{ \pm}$if $v \notin \sigma$ and $\psi_{\sigma}\left(v^{ \pm}\right)=v^{+}$if $v \in \sigma$.

Let $\iota_{\sigma}$ denote the inclusion $\left\{S_{L}-N b^{1}\left(\sigma, S_{L}\right)\right\} \hookrightarrow S_{L}$. Note: $\psi_{\sigma} \circ \iota_{\sigma}$ is the identity map from $\left\{S_{L}-N b^{1}\left(\sigma, S_{L}\right)\right\}$ to itself. Thus the map $\left(\iota_{\sigma}\right)_{*}: \pi_{1}\left(S_{L}-N b^{1}\left(\sigma, S_{L}\right)\right) \rightarrow$ $\pi_{1}\left(S_{L}\right)$ and the maps $\left(\iota_{\sigma}\right)_{\sharp}: H_{m}\left(S_{L}-N b^{1}\left(\sigma, S_{L}\right)\right) \rightarrow H_{m}\left(S_{L}\right)$ are injective.

Remark 4.4. Proposition 4.1 can also be viewed from a Morse theory perspective, similar to that in $[\mathrm{BB}$. Bestvina uses such an approach in describing the topology at infinity for Artin groups of finite type [B].

In our case, a Morse function is given by the distance from the base vertex $v \in X$. Note that Lemma 2.2 ensures that the only cells on which this function is constant are the vertices of $X$, and so it has isolated critical points. The ascending links are precisely the $O p(w, v)$ for a vertex $w \neq v$, and the ascending link of $v$ is all of $L k(v, X)$. As the radius of $B_{r}(v)$ decreases to 0 , the topology of $X-B_{r}(v)$ can change only when $S_{r}(v)$ passes through vertices of $X$. When $r=0$, we obtain the contractible space $X$. Thus, if $L k(v, X)$ and the $O p(w, v)$ are all $m$-connected (resp. $m$-acyclic), then the compliments $X-B_{r}(v)$ are also $m$-connected (resp. $m$-acyclic). Hence, $X$ is $m$-connected (resp. $m$-acyclic) at infinity.

Question 1. Can one give a simple description of the connectivity at infinity properties for arbitrary Artin groups? We note that the homology at infinity for arbitrary Coxeter groups has been computed in [Da 2].

\section{Duality}

Here we sketch the proof of our characterization of the topology of $S_{L}$ in terms of the topology of $L$, along with the proof of Theorem $\mathrm{C}$ from the introduction.

Lemma 5.1. The link $S_{L}$ is $m$-acyclic if and only if $L$ is $m$-acyclic, and for any simplex $\sigma \subset L$, the link of $\sigma$ in $L$ is $(m-d(\sigma)-1)$-acyclic. The link of a vertex in $\widetilde{K_{L}}$ is $m$-connected if, in addition to the conditions above, $L$ is simply connected.

Combining this result with Theorem B gives us the following corollary.

Corollary 5.2. Let $L$ be a finite flag complex which is different from the 0- or 1-simplex. Then the right angled Artin group $A_{L}$ is simply connected at infinity if and only if $L$ is simply connected and contains no cut vertex.

We establish some notation for various simplicial maps from $S_{L}$ to itself which will be used in the proof of Lemma 5.1. We define these maps on the level of $S_{L}^{(0)}$, and extend simplicially. The map $\phi_{i}: S_{L} \rightarrow S_{L}$ takes $v_{i}^{-}$to $v_{i}^{+}$and leaves all the other vertices fixed. If $\sigma$ is a simplex of $L, \Phi_{\sigma}$ is the composition of the $\phi_{i}$ 's for 
all $v_{i} \notin \sigma$. We denote $\Phi_{\emptyset}$ by $\Phi$; this is the same map $\Phi$ which we used in $\S 3$. If we let $\iota_{\sigma}: \Phi_{\sigma}\left(S_{L}\right) \hookrightarrow S_{L}$ denote the inclusion map, then $\Phi_{\sigma}\left(S_{L}\right) \stackrel{\iota_{\sigma}}{\hookrightarrow} S_{L} \stackrel{\Phi_{\sigma}}{\longrightarrow} \Phi_{\sigma}\left(S_{L}\right)$ is the identity on $\Phi_{\sigma}\left(S_{L}\right)$. Hence $\left(i d_{\Phi_{\sigma}\left(S_{L}\right)}\right)_{*}=\left(\phi_{\sigma}\right)_{*} \circ\left(\iota_{\sigma}\right)_{*}$ and in particular $\left(\iota_{\sigma}\right)_{*}$ is injective.

Proof. We first establish the base case, in the context of simple connectivity.

$(\Leftarrow)$ Since $S_{L}$ is simplicial, we can describe any closed edge path by the vertices it passes through: $\left[v_{0}^{\varepsilon_{0}}, v_{1}^{\varepsilon_{1}}, \ldots, v_{k}^{\varepsilon_{k}}\right]$. Here we are keeping the notation simple; it's possible that $v_{i}=v_{j}$ for $i \neq j$. Below we construct a free homotopy from this edge path to the path described by $\left[v_{0}^{+}, v_{1}^{+}, \ldots, v_{k}^{+}\right]$. Since this second path is contained in a copy of $L$, and $L$ is simply-connected, there is a homotopy contracting this loop to a point.

Given an index $j$ such that $\varepsilon_{j}=-$, we show that the subpath $\left[v_{j-1}^{\varepsilon_{j-1}}, v_{j}^{-}, v_{j+1}^{\varepsilon_{j+1}}\right]$ is homotopic (relative to the end points) to the path $\left[v_{j-1}^{\varepsilon_{j-1}}, v_{j}^{+}, v_{j+1}^{\varepsilon_{j+1}}\right]$.

Case (i): $v_{j-1}^{\varepsilon_{j-1}}=v_{j+1}^{\varepsilon_{j+1}}$. Here the argument is trivial; one has a redundant edge that can be replaced by any other redundant edge.

Case (ii): $v_{j-1}=v_{j+1}$ but $\varepsilon_{j-1} \neq \varepsilon_{j+1}$. In this case use the fact that the edge $\left[v_{j-1}, v_{j}\right]$ has a non-empty link in $L$ to find a vertex $w \in L$ which is adjacent to both $v_{j-1}$ and $v_{j}$. Using the 2 -sphere in $S_{L}$ determined by the triple $\left\{v_{j-1}, v_{j}, w\right\}$ one can homotope $\left[v_{j-1}^{\varepsilon_{j-1}}, v_{j}^{-}, v_{j+1}^{\varepsilon_{j+1}}\right]$ to $\left[v_{j-1}^{\varepsilon_{j-1}}, v_{j}^{+}, v_{j+1}^{\varepsilon_{j+1}}\right]$ (relative to the end points).

Case (iii): $v_{j-1} \neq v_{j+1}$. Since $L k\left(v_{j}, L\right)$ is connected, there is an edge path

$$
\left[v_{j-1}, w_{1}, w_{2}, \ldots, w_{l}, v_{j+1}\right] \subset L k\left(v_{j}, L\right)
$$

this path describes an edge path

$$
\left[v_{j-1}^{\varepsilon_{j-1}}, w_{1}^{+}, w_{2}^{+}, \ldots, w_{l}^{+}, v_{j+1}^{\varepsilon_{j+1}}\right]
$$

which is contained both in $L k\left(v_{j}^{-}, S_{L}\right)$ and in $L k\left(v_{j}^{+}, S_{L}\right)$. Hence the suspension of this path, with suspension points $v_{j}^{-}$and $v_{j}^{+}$, is contained in $S_{L}$, so $\left[v_{j-1}^{\varepsilon_{j-1}}, v_{j}^{-}, v_{j+1}^{\varepsilon_{j+1}}\right]$ $\simeq\left[v_{j-1}^{\varepsilon_{j-1}}, v_{j}^{+}, v_{j+1}^{\varepsilon_{j+1}}\right]$.

$(\Rightarrow)$ : To establish the converse, first consider the possibility that $L$ is not simplyconnected. Then because $\iota: L \hookrightarrow S_{L}$ is $\pi_{1}$-injective, $\pi_{1}\left(S_{L}\right) \neq\{1\}$. Similarly if the link of a vertex $v$ is not connected, but $L$ is simply connected, it follows by Mayer-Vietoris that $v$ is a cut vertex of $L$. Hence the pair of vertices $\left\{v^{-}, v^{+}\right\}$ separates $S_{L}$. But then by a generalized van Kampen's theorem, $\pi_{1}\left(S_{L}\right) \neq\{1\}$. Finally, if the link of some edge $[v, w] \subset L$ is empty, then either $L=[v, w]$, or the link of $v$ or $w$ is not connected and we are in the previous case.

We now turn to the general case, arguing by induction.

$(\Leftarrow)$ Our plan is similar to what we did in the base case: Take an $m$-cycle $z$ in $S_{L}$, and show that this is homologous to an $m$-cycle $z^{\prime}$ whose support is in $\iota(L)$. At that point we will be done since $L$ is assumed to be $m$-acyclic. In our argument we will frequently refer to the fact that $L k\left(v^{-}, S_{L}\right)=L k\left(v^{+}, S_{L}\right) \simeq S_{L k(v, L)}$.

If $\sigma \subset L k(v, L)$, then $L k(\sigma, L k(v, L)) \simeq L k(\sigma *\{v\}, L)$ and hence by hypothesis, $\operatorname{Lk}(\sigma, L k(v, L))$ is $(m-d(\sigma *\{v\})-1)$-acyclic, and therefore it is $((m-1)-d(\sigma)-1)$ acyclic. It follows by induction that $S_{L k(v, L)}$ is $(m-1)$-acyclic. Since $S_{S t(v, L)}$ is the suspension of $S_{L k(v, L)}$ with suspension points $v^{-}$and $v^{+}, S_{S t(v, L)}$ is $m$-acyclic. Notice that the map $\phi_{i}: S_{L} \rightarrow S_{L}$ induces a map $\left(\phi_{i}\right)_{\sharp}$ on the chain level. The 
difference $z-\left(\phi_{i}\right)_{\sharp}(z)$ is an $m$-cycle contained in $S_{S t(v, L)}$, hence $z$ is homologous to $\left(\phi_{i}\right)_{\sharp}(z)$. It follows that $z$ is homologous to $\Phi(z) \subset \iota(L)$.

$(\Rightarrow)$ As in the base case, if $L$ is not $m$-acyclic, then $S_{L}$ is not $m$-acyclic because the induced map on homology $\iota_{\sharp}$ is injective. Consider then the possibility that $L$ is $m$-acyclic but $L k(v, L)$ is not $(m-1)$-acyclic for some vertex $v$. Notice that $\Phi_{v}\left(S_{L}\right)$ can be expressed as the union of a copy of $L$ with a copy of $\left\{v^{-}\right\} * L k(v, L)$ with intersection $L k(v, L)$. By Mayer-Vietoris $H_{m}\left(\Phi_{v}\left(S_{L}\right)\right) \simeq H_{m-1}(L k(v, L)) \neq 0$. Since the induced map on homology $\left(\iota_{v}\right)_{\sharp}$ is injective, it follows that $H_{m}\left(S_{L}\right) \neq 0$.

Finally, assume that for all simplices $\tau$ of dimension less than $k$ that $L k(\tau, L)$ is $(m-d(\tau)-1)$-acyclic, but there is some $k$-simplex $\sigma$ where $L k(\sigma, L)$ is not $(m-k-1)$-acyclic. Let $\sigma^{(0)}=\left\{w_{0}, \ldots, w_{k}\right\}$ and let $\sigma^{-}$denote the copy of $\sigma$ in $\Phi_{\sigma}\left(S_{L}\right)$ whose vertex set is $\left\{w_{0}^{-}, \ldots, w_{k}^{-}\right\}$. Note that $\Phi_{\sigma}\left(S_{L}\right)$ is a flag complex, so $N b^{1}\left(\sigma^{-}, \Phi_{\sigma}\left(S_{L}\right)\right)$ is contractible. Further, $\Phi_{\sigma}\left(S_{L}\right)$ can be expressed as the union of a copy of $L$ and $N b^{1}\left(\sigma^{-}, \Phi_{\sigma}\left(S_{L}\right)\right)$. Again, by Mayer-Vietoris, $H_{m}\left(\Phi_{\sigma}\left(S_{L}\right)\right) \simeq$ $H_{m-1}(I)$ where $I$ is the intersection. Again since the homology of $\Phi_{\sigma}\left(S_{L}\right)$ injects into the homology of $S_{L}$, it suffices to show that $H_{m-1}(I) \neq 0$. But the intersection $I$ can be expressed as the union of the $L k\left(w_{i}, L\right)$, where for any $J \subset\{0,1, \ldots, k\}$, the intersection $\bigcap_{i \in J} L k\left(w_{i}, L\right) \simeq L k\left(\sigma_{J}, L\right)$. Decomposing $I$ as

$$
I=L k\left(w_{0}, L\right) \cup\left\{\bigcup_{i \geq 1} L k\left(w_{i}, L\right)\right\}
$$

and again applying Mayor-Vietoris achieves the desired result.

We now establish Theorem $\mathrm{C}$ from the introduction.

An $F P_{\infty}$ group $G$ is a duality group if $H^{i}(G, \mathbb{Z} G)=0$ for $i \neq n$ and $H^{n}(G, \mathbb{Z} G)$ is torsion free. One can show that $G$ is $(n-2)$-acyclic at infinity if and only if $H^{i}(G, \mathbb{Z} G)=0$ for $i \leq n-1$ and $H^{n}(G, \mathbb{Z} G)$ is torsion free (see $\S 4.3$ and $\S 5.5$ in $[\mathrm{G}])$. Further, $G$ has cohomological dimension $n$ if and only if $H^{i}(G, \mathbb{Z} G)=0$ for $i>n$ and $H^{n}(G, \mathbb{Z} G) \neq 0$ (see $[\mathrm{B}]$ ). Hence $G$ is a duality group if and only if $c d(G)=n$ and $G$ is $(n-2)$-acyclic at infinity.

It is easy to establish that the cohomological dimension of $A_{L}$ is the dimension of the Eilenberg-Mac Lane space $K_{L}$. So by Theorem B $A_{L}$ is a duality group if and only if $\widetilde{K_{L}}$ is $\left(\left|S_{L}\right|-2\right)$-acyclic at infinity. But by Lemma 5.1 , this can happen if and only if $L$ is Cohen-Macaulay. The 'Cohen-Macaulay' terminology was pointed out to us by Mike Davis, who also asked the following question, which is closely related to Question 1.

Question 2. (M. Davis) Is a similar result true for arbitrary Artin groups? That is, is an Artin group a duality group if and only if the link of each vertex in the Salvetti complex is Cohen-Macaulay?

Remark 5.3. See $\mathrm{BM}$ for recent progress on Questions 1 and 2.

\section{Applichtions to group extensions}

Stallings showed that if

$$
1 \rightarrow K \rightarrow G \rightarrow L \rightarrow 1
$$

is a short exact sequence of finitely generated infinite groups, then $G$ is one-ended (=0-connected at infinity). Houghton and Jackson independently established that $G$ is simply-connected at infinity when all the groups in the short exact sequence 
are finitely presented and either $K$ or $L$ is one-ended. In [G], Ross Geoghegan has proven the following generalization.

Theorem 6.1. (Geoghegan [G]) Let $1 \rightarrow K \rightarrow G \rightarrow L \rightarrow 1$ be a short exact sequence of infinite groups all of type $F_{m+1}$. Let $K$ be $k$-acyclic at infinity and let $L$ be l-acyclic at infinity (Z्Z-coefficients) where $k$ and $l$ are at most $m$. Let $u=\min (k+l+2, m)$. Then $G$ is u-acyclic at infinity. If $k$ or $l$ is at least 0 (that is, one of $K$ or $L$ is one-ended), then $G$ is u-connected at infinity.

A similar theorem, using semistability, can be found in print in GM 1. We highlight one specific case.

Corollary 6.2. Let $1 \rightarrow K \rightarrow G \rightarrow L \rightarrow 1$ be a short exact sequence of infinite groups all of type $F_{m+1}$, where $m \geq 1$. Let $L$ be $(m-1)$-acyclic at infinity ( $\mathbb{Z}$ coefficients). Then $G$ is $m$-connected at infinity.

Because it is difficult to establish finiteness properties of infinite index subgroups, it's important to find minimal finiteness hypotheses on $K$ which are necessary to establish connectivity at infinity properties for $G$. For example, the kernel is not assumed to have any connectivity at infinity properties in Corollary 6.2. As a matter of fact, the quotient will be $F_{m+1}$ even if the kernel is only $F_{m}$, so requiring the kernel to be $F_{m+1}$ might appear to be stronger than would be absolutely necessary.

Mihalik has recently shown that one can't get rid of the hypothesis that the kernel is finitely presented in the Houghton/Jackson result, even if you improve the connectivity at infinity of the quotient [Mi 2]. Here we show how Mihalik's example can be extended to show that one can't assume the kernel is $F_{m}$ in Corollary 6.2; that is, our examples show that Geoghegan's result is optimal.

We use a "blowing up" process. For those familiar with the terminology, if $A_{L}$ is a right angled Artin group, the $n$-blow up of $A_{L}$ is the graph product based on $L^{(1)}$ where each vertex group is $\simeq \mathbb{Z}^{n}$. Equivalently one can form a new complex from $L$ where for each vertex $v_{i}$ takes a collection of $n$ vertices $\left\{v_{i, 1}, \ldots v_{i, n}\right\}$ and connect $v_{i, k}$ to $v_{j, l}$ whenever $i=j$ or $v_{i}$ was connected to $v_{j}$ in $L$. This process has the effect that each $(m-1)$-simplex of $L$ is replaced by an $(m n-1)$-simplex, and we denote the new complex by $\beta_{n}(L)$. Since $\beta_{n}(L)$ deformation retracts onto $L$, the homology and homotopy groups of $\beta_{n}(L)$ are isomorphic to the homology and homotopy groups of $L$.

First Examples. Here we construct examples showing that the $F_{m+1}$ condition in Corollary 6.2 cannot be weakened to $F_{m}$. Let $L$ be an $m$-octahedron and let $n>m+1$. Let $\phi: A_{\beta_{n}(L)} \rightarrow \mathbb{Z}^{m+1} \simeq\left\langle x_{1}\right\rangle \times \cdots \times\left\langle x_{m+1}\right\rangle$ via $\phi\left(v_{i, k}\right)=x_{k}$ for $k \leq m+1$ and $\phi\left(v_{i, k}\right)=0$ for $k>m+1$.

The quotient $\mathbb{Z}^{m+1}$ is $(m-1)$-connected at infinity.

Using the Main Theorem of MMV one can show that the kernel is $F_{m}$ but not $F_{m+1}$. Further, let $\phi^{\prime}$ be the map from $A_{\beta_{n}(L)}$ onto $\mathbb{Z}^{n}$ defined by $\phi^{\prime}\left(v_{i, k}\right)=x_{k}$. Denote the kernel of this map by $K^{\prime}$. Then there is a short exact sequence

$$
1 \rightarrow K^{\prime} \rightarrow K \rightarrow \mathbb{Z}^{n-m-1} \rightarrow 1
$$

Again by [MM], $K^{\prime}$ is of type $F_{m}$, hence by Corollary 6.2 above, if we chose $n \geq 2 m+1$, we know that the kernel $K$ is $(m-1)$-connected at infinity.

However, although the kernel and quotient can be arranged to be $(m-1)$ connected at infinity, the right angled Artin group $A_{\beta_{n}(L)}$ is not $m$-connected at infinity by Theorem B and Lemma 5.1. 


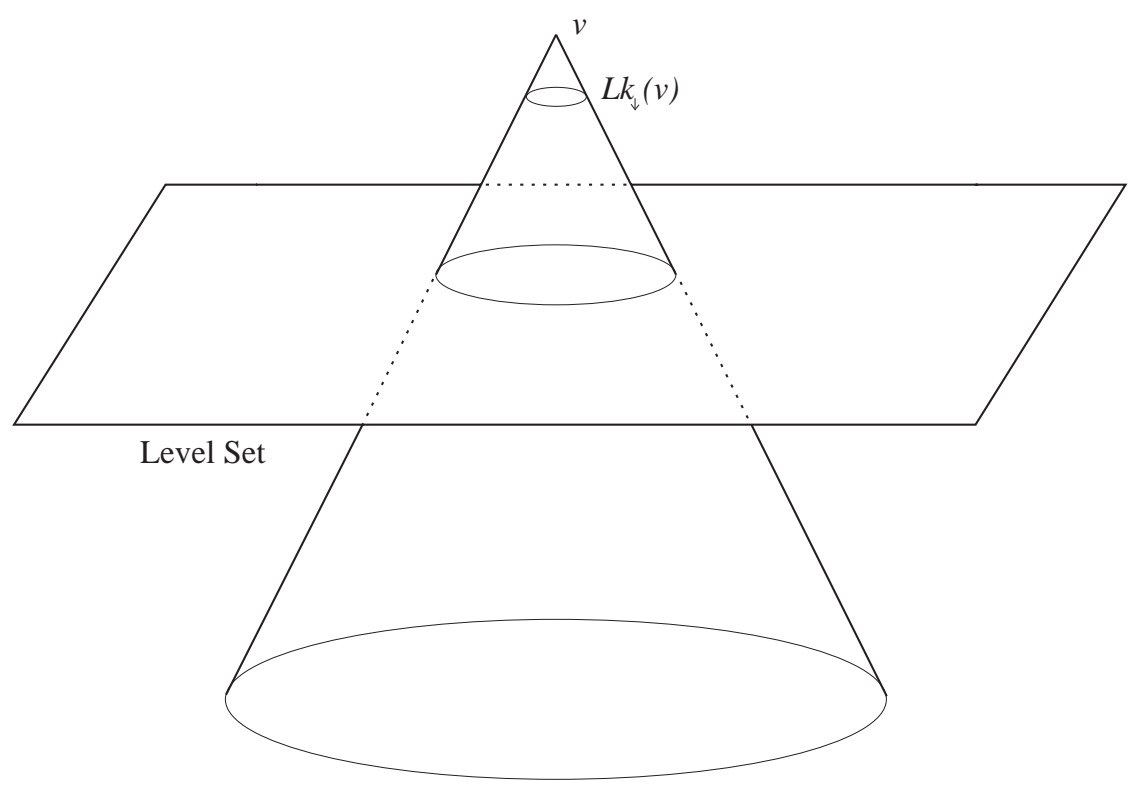

Figure 3. The common source of obstruction to finiteness in $K$ and connectivity at infinity in $\widehat{K_{L}}$

Second Examples. Let $L$ be the second barycentric subdivision of the presentation 2-complex associated to Higman's group

$$
G=\left\langle x, y, z, w \mid x^{y}=x^{2}, y^{z}=y^{2}, z^{w}=z^{2}, w^{x}=w^{2}\right\rangle .
$$

It follows that $L$ is a flag complex with trivial homology in all dimensions but nontrivial $\pi_{1}$. Form the right angled Artin group $A_{\beta_{n}(L)}$ and let $\phi: A_{\beta_{n}(L)} \rightarrow \mathbb{Z}^{m}$ for $n>m$ as above. Again one can use [MMV] to show that the kernel of this map is $F P_{\infty}$ but it is not finitely presented. By choosing $n>2 m>0$ one creates a short exact sequence $1 \rightarrow K \rightarrow G \rightarrow \mathbb{Z}^{m} \rightarrow 1$ where: the rank of the quotient can be made arbitrarily large; the group $G$ is not simply connected at infinity; the kernel is $F P_{\infty}$ and can be made $n$-acyclic at infinity for any $n$. Thus homological finiteness conditions on the kernel, even combined with homological connectivity at infinity, are never enough to insure the Houghton/Jackson conclusion that the extension is simply connected at infinity. You simply have to require that the kernel is finitely presented.

Comment. We note that the obstruction to finiteness properties for the kernels is exactly the same obstruction that forces limited connectivity at infinity. These kernels act on level sets inside of $\widetilde{K_{L}}$. The techniques of $[\mathrm{BB}]$ show that the reason these kernel's finiteness properties are bounded by the topology of $L$ is because non-trivial $\pi_{m}$ in $L$ injects into $S_{L}$ and projects onto the level sets that the kernels act on. Our arguments in this paper establish that this non-trivial $\pi_{m}$ also projects out to infinity. 


\section{REFERENCES}

[B] M. Bestvina, Nonpositively curved aspects of Artin groups of finite type, Geometry and Topology 3 (1999), 269-302. MR 2000h:20079

[BB] M. Bestvina and N. Brady, Morse theory and finiteness properties of groups, Invent. Math. 129 (1997), 445-470. MR 98i:20039

[BF] M. Bestvina and M. Feighn, The topology at infinity of $\operatorname{Out}\left(F_{n}\right)$, Invent. Math. 140 (2000), 651-692.

[BH] M. Bridson and A. Haefliger, Metric spaces of non-positive curvature, manuscript of a book, in progress.

[BT] M. G. Brin and T. L. Thickstun, 3-manifolds which are end 1-movable, Mem. Amer. Math. Soc. 81 no. 411 (1989). MR 90g:57015

[Br] K. Brown, Cohomology of Groups, vol. GTM 87, Springer-Verlag, New York, 1982. MR 83k:20002

[BM] K.S. Brown and J. Meier, Improper actions and higher connectivity at infinity, Comment. Math. Helv. 75 (2000), 171-188. CMP 2000:13

[Da 1] M. W. Davis, Groups generated by reflections and aspherical manifolds not covered by Euclidean space, Ann. Math. 117 (1987), 293-324. MR 86d:57025

[Da 2] M. W. Davis, The cohomology of a Coxeter group with group ring coefficients, Duke Math. Jour 91 (1998), 297-314. CMP 99:06; MR 99b:20067

[G] R. Geoghegan, Topological Methods in Group Theory, manuscript of a book, in progress.

[GM 1] R. Geoghegan and M. L. Mihalik, Free abelian cohomology of groups and ends of universal covers, J. Pure Appl. Algebra 36 (1985), 123-137. MR 86h:20074

[GM 2] R. Geoghegan and M. L. Mihalik, The Fundamental Group at Infinity, Topology 35 (1996), 655-669. MR 97h:57002

[H] C. H. Houghton, Cohomology and the behavior at infinity of finitely presented groups, J. Lond. Math. Soc. (2) 15 (1977), 465-471. MR 56:15782

$[\mathrm{J}] \quad$ B. Jackson, End invariants of group extensions, Topology 21 (1982), 71-81. MR 83a:57002

[MS] S. Mardešić and J. Segal, Shape Theory, North-Holland, (1982). MR 84b:55020

[Me] J. Meier, Geometric invariants for Artin groups, Proc. London Math. Soc. (3) 74 (1997), 151-173. MR 97h:20049

[MMV] J. Meier, H. Meinert and L. VanWyk, Higher generation subgroup sets and the $\Sigma$ invariants of graph groups, Comment. Math. Helv 73 (1998), 22-44. MR 99f:57002

[MV] J. Meier and L. VanWyk, The Bieri-Neumann-Strebel invariants for graph groups, Proc. London Math. Soc. (3) 71 (1995), 263-280. MR 96h:20093

[Mi 1] M. L. Mihalik, Semistability at the end of a group extension, Trans. Amer. Math. Soc. 277 (1983), 307-321. MR 84d:57001

[Mi 2] M. L. Mihalik, Semistability of Artin and Coxeter groups, J. Pure Appl. Algebra 111 (1996), 205-211. MR 97e:20060

[Mi 3] M. L. Mihalik, Semistability at infinity, simple connectivity at infinity, and normal subgroups, Top. Appl. 72 (1996), 273-281. MR 97j:20035

[P] J. Profio, Using subnormality to show the simple connectivity at infinity of a finitely presented group, Trans. Amer. Math. Soc. 320 (1990), 218-232. MR 90k:20057

[R] J. P. Rickert, A proof of the simple connectivity at infinity of Out $\left(F_{4}\right)$, J. Pure Appl. Algebra 145 (2000), 59-73. CMP 2000:06

[St] J. R. Stallings, On torsion free groups with infinitely many ends, Ann. of Math. 88 (1968), 312-334. MR 37:4153

[Sr] R. Strebel, A remark on subgroups of infinite index in Poincaré duality groups, Comment. Math. Helv. 52 (1977), 317-324. MR 56:15793

[V] K. Vogtmann, End invariants of the group of outer automorphisms of a free group, Topology 34 (1995), 533-545. MR 96h:20068

Department of Mathematics, University of Oklahoma, Norman, Oklahoma 73019

E-mail address: nbrady@math.ou.edu

Department of Mathematics, Lafayette College, Easton, Pennsylvania 18042

E-mail address: meierj@lafayette.edu 